\title{
FUNDAMENTOS EN SISTEMAS DE EVALUACIÓN DE LA EDUCACIÓN SUPERIOR: BRASIL, COLOMBIA Y ARGENTINA
}

\author{
NINA ELEONOR VIZCARRA HERLES, ELISABETE \\ MONTEIRO DE AGUIAR PEREIRA
}

\section{RESUMO}

A avaliação é, ao mesmo tempo, uma questão de urgência e complexa, devido à demanda da sociedade pela melhoria de suas instituições e organizações. O tema de pesquisa do presente trabalho pertence ao campo da avaliação da educação superior, o objeto de análises são os fundamentos conceituais da avaliação e sua presença na concepção dos Sistemas da Avaliação para a Educação Superior, levando em conta os casos de Brasil, Colômbia e Argentina. Este artigo pretende organizar um marco histórico referencial sobre a evolução dos fundamentos da avaliação, desde a avaliação da aprendizagem até a avaliação institucional; baseado nos aportes de Tyler, Crombach, Scriven, Guba y Lincoln, House, e Stufflebeam, e de autores europeus e latino americanos; para buscar a referência teórica das concepções que fundamentam os sistemas de avaliação. Finalmente são analisadas e comparadas as características das bases conceituais dos sistemas de avaliação do ensino superior acima mencionados. 


\section{RESUMEN}

La evaluación es, al mismo tiempo, una cuestión de urgencia y compleja, debido a la demanda de la sociedad por la mejora de sus instituciones y organizaciones. El tema de investigación de este trabajo pertenece al campo de la evaluación de la educación superior. El objeto de análisis son los fundamentos conceptuales de la evaluación y su presencia en la concepción de los Sistemas de Evaluación para la Educación Superior, teniendo en cuenta los casos de Brasil, Colombia, y Argentina. Este artículo tiene la intención de organizar un marco histórico referencial sobre la evolución de los fundamentos de la evaluación, desde la evaluación del aprendizaje hasta la evaluación institucional, basado en las contribuciones de Tyler, Cronbach, Scriven, Guba y Lincoln, House, Stufflebeam, y de autores europeos y latinoamericanos; para luego buscar la referencia teórica de las concepciones que fundamentan los sistemas de evaluación. Finalmente, se analizan y comparan las características de las bases conceptuales de los sistemas de evaluación de la enseñanza superior antes mencionados.

PALABRAS CLAVE: EVALUACIÓN DE LA EDUCACIÓN •

ENSEÑANZA SUPERIOR • ANÁLISIS DE SISTEMAS • SISTEMAS DE EVALUACIÓN.

\section{ABSTRACT}

Assessment is at the same time an urgent and complex matter due to society's demands to improve their institutions and organizations. The research topic of this study belongs to the field of evaluation of higher education, the object of analysis are the conceptual foundations of evaluation and their presence in the design of Assessment Systems for Higher Education, taking into account the cases of Brazil, Colombia and Argentina. This article intends to organize a landmark reference of the evolution of the foundations of assessment, from assessment of learning up to institutional evaluation, based on contributions from Tyler, Cronbach, Scriven, Guba y Lincoln, House, and Stufflebeam, as well as European and Latin Americans authors, to seek the reference of theoretical concepts that underlie the assessment systems. Finally, we analyzed and compared the characteristics of the conceptual basis of the higher education assessment systems mentioned above.

KEYWORDS: ASSESSMENT OF EDUCATION • HIGHER

EDUCATION • SYSTEMS ANALYSIS • ASSESSMENT SYSTEMS. 


\section{INTRODUCCIÓN}

La evaluación es un concepto que ha evolucionado con el tiempo avanzando en el camino del desarrollo de la ciencia y la tecnología. A finales de siglo pasado en las propuestas organizacionales se hacía referencia a un modelo de sistemas y en este siglo ya se habla de un modelo post sistémico o inteligente, según el cual la evaluación se constituye ahora en el componente inteligente de una organización.

La evaluación encierra en sí la búsqueda del cambio hacia lo que para nosotros significa la mejora de la calidad en la educación, de manera que sea propia de nuestro contexto y que satisfaga las expectativas y necesidades de toda la sociedad. Por lo que es necesario tener en cuenta que si reformamos nuestros sistemas, estos no debieran cimentarse bajo una concepción mercantilista o clientelista de la educación, que contribuya a la formación de profesionales con poca sensibilidad social, que constituyan parte de las cadenas de producción para los mercados, pues en consecuencia se estaría contribuyendo a la formación de una sociedad irreflexiva, amoral, destructiva, acrítica y no creativa en los aportes a la solución de los problemas que plantea el desarrollo. 
La evaluación muchas veces despierta objeciones sobre las concepciones que se toman para evaluar, así como en relación a los agentes evaluadores, demandando que el sistema de evaluación por lógica deba tener coherencia, consistencia y congruencia para validarse por sí mismo, en vista de que la evaluación pretende encontrar elementos que nos llevarán hacia el perfeccionamiento de la institución u organización.

En el campo de la evaluación de la educación se generan muchos debates, entre ellos tenemos, si la evaluación responde a una concepción conductista (behaviorista) o a una constructivista, si la práctica de la evaluación es coherente con las propuestas teóricas, si en la evaluación son mas importantes los insumos, los procesos o los resultados, si el paradigma adecuado en la evaluación institucional y de programas es el cuantitativo o el cualitativo, si la función de la evaluación es regulatoria o formativa, etc.

Por ello adquiere relevancia la revisión de las concepciones de la evaluación que fundamentan los sistemas de evaluación institucional y de programas para comprender su funcionamiento y desarrollo.

\section{METODOLOGÍA}

A. Se realizó un análisis documental de artículos publicados y documentos de organismos gubernamentales, de Colombia: Consejo Nacional de Acreditación (CNA), Ministerio de Educación de Colombia; de Brasil: Comisión Nacional de Evaluación de la Educación Superior (Conaes), Sistema Nacional de Evaluación de la Educación Superior (Sinaes), Instituto Nacional de Estudios y Pesquisas Educacionales Anísio Teixeira (Inep) y Ministerio de Educación de Brasil; de Argentina: Comisión Nacional de Acreditación (Coneau), Consejo Interuniversitario Nacional (CIN). Se utilizaron como categorías de análisis: las concepciones y fundamentos de evaluación, identificándose la afinidad entre los contenidos conceptuales presentes en las concepciones de evaluación de los diversos autores y los contenidos conceptuales presentes en las concepciones 
y fundamentos de evaluación presentes en las propuestas de los sistemas de evaluación de la educación superior.

B. El análisis comparativo de las concepciones de evaluación de los sistemas de evaluación en los casos de Brasil, Colombia y Argentina, se realizó siguiendo el esquema propuesto por G. F. Bereday (1968) y F. Hilker, (1964) citados por Ferrán (2002), el cual considera las siguientes operaciones:

a. Descripción - para obtener y ordenar la información que interesa.

b. Interpretación - para decodificar e internalizar los datos y explicar la información.

c. Yuxtaposición - para confrontar los contenidos de la información.

d. Comparación - para examinar semejanzas y diferencias mediante el análisis de la información, llegando a las conclusiones que correspondan.

\section{RESULTADOS Y ANÁLISIS}

3.1 FUNDAMENTOS DE LA EVALUACIÓN: DESDE LA EVALUACIÓN DEL APRENDIZAJE A LA EVALUACIÓN INSTITUCIONAL

En la historia de la evaluación en el ámbito de la educación, las contribuciones de Ralph Tyler han sido importantes para el desarrollo de este concepto. Considerado padre de la evaluación educacional debido a la investigación realizada entre 1930 y 1940, Tyler se interesó por hallar evidencias válidas para evaluar el comportamiento que expresa el progreso de los estudiantes hacia los objetivos de aprendizaje, para Tyler, según Pinar et al. (1995) la evaluación es un proceso para determinar hasta qué punto los objetivos educacionales fueron realmente alcanzados, por lo tanto, si los instrumentos de evaluación nos permiten ubicar las evidencias válidas sobre el progreso de los estudiantes hacia los objetivos educacionales, entonces estos instrumentos son los más pertinentes en el trabajo de evaluación, las técnicas de evaluación debe estar en correspondencia con la clase de comportamiento a ser evaluado.

Más adelante en su obra de 83 páginas "Basic Principles of Curriculum and Instruction”, Tyler (1949) propone cuatro principios básicos para la evaluación del aprendizaje:

1. Definición de objetivos de aprendizaje apropiados. 
2. El establecimiento de útiles experiencias de aprendizaje.

3. Organizar las experiencias de aprendizaje que tienen un efecto acumulativo máximo.

4. La evaluación de los planes de estudio y la revisión de sus aspectos

Tyler propone también cuatro principios para la evaluación del currículo que están en relación con las siguientes interrogantes:

\section{¿Cuáles son los objetivos deseados? \\ ¿Con qué actividades pueden ser alcanzados? \\ ¿Cómo pueden estas experiencias organizarse de manera eficiente? \\ ¿Cómo puede probarse que se alcanzarán los objetivos? (apud Escudero, 2003, p. 5)}

Si observamos detalladamente las conclusiones arriba encontraremos el propósito del pensamiento de Tyler sobre la importancia de los objetivos y de las técnicas e instrumentos para evaluar el comportamiento de los estudiantes en el proceso de enseñanza. Tyler afirma que los objetivos de aprendizaje deben ser claramente definidos, los instrumentos deben proporcionar evidencias válidas y las técnicas deben ser apropiadas al comportamiento a ser evaluado. Estos aspectos a través de los años se fueron revisando y reconceptualizando en los diferentes modelos y enfoques de evaluación.

Ralph Tyler presenta para su tiempo, una renovada visión de la evaluación del currículo y de la evaluación del aprendizaje. Plantea el modelo de planificación curricular en el nivel tecnológico, en el cual pone énfasis en la selección y organización del contenido, así como en las estrategias para transmitir la información y evaluar el logro de los objetivos de aprendizaje.

Para Tyler, la evaluación determina en qué medida han sido alcanzados los objetivos y para ello, sugiere realizar comparaciones entre los resultados y los objetivos propuestos en un programa educativo.

La evaluación del aprendizaje años más tarde evoluciona recogiendo contribuciones que se plantean desde el punto de vista de la sociología de la educación, conforme es mencionado por Broadfoot: 
Uma sociologia da avaliação deve abranger toda a variedade de atividades avaliativas no contexto do sistema educativo, desde os julgamentos que os professores fazem sobre cada aluno na sala de aula, até aos vários e mais formais modos de julgar o progresso dos alunos na escola como um todo, que possibilitam a responsabilização do professor, da escola, das autoridades locais e, em última instância, de todas as formas de provisão da educação a nível nacional (Broadfoot, 1979, p. 11)

Esta concepción nos deja ver una cuestión importante, en la práctica de la evaluación del aprendizaje de los alumnos, el juzgamiento de los profesores sobre el progreso de cada alumno en el aula, es sólo una parte de la evaluación siendo importante también juzgar el progreso de los alumnos en la escuela como un todo, este aspecto involucra la evaluación de niveles de socialización del estudiante donde pueden ser observadas además la práctica de actitudes y el cultivo de valores.

Otro aporte a la evaluación del aprendizaje es desde la perspectiva de la filosofía de la evaluación, en los años 1980 y 1990, que se expresa en los cuestionamientos: “¿Qué es lo que se tiene que enseñar en el aula? -y por lo tanto- ¿Qué es lo que se tiene que evaluar?" (Melo, 2004, P. 182).

Estas sencillas interrogantes hacen referencia a aspectos importantes de la educación, como son los contenidos y los propósitos de la enseñanza, sin embargo su respuesta tiene repercusiones en el carácter de la educación y en el carácter de la sociedad, que afectan los propósitos de la evaluación.

La evaluación del aprendizaje evolucionó también hacia otros paradigmas, como la concepción que plantea la evaluación bajo el paradigma cognitivo. Castro O. (1999), menciona entre las características de este enfoque de evaluación las siguientes:

- La subordinación de la enseñanza al aprendizaje.

- El protagonismo fluctuante del maestro y los estudiantes.

- La diversificación de los roles del maestro en la intervención, medición, coordinación y facilitación del aprendizaje de los alumnos.

- La evaluación basada en este paradigma debe estar orientada a la valoración, al análisis cualitativo de los procesos, sus estadios intermedios y los productos, 
con una inspiración crítica y una finalidad formativa, educativa.

Este enfoque privilegia el papel activo del alumno en el aprendizaje y promueve la evaluación formativa. A diferencia de la propuesta de Tyler que prioriza la comparación de resultados con objetivos propuestos, en esta propuesta son importantes los factores que intervienen en el proceso de aprendizaje para lograr el desarrollo de habilidades cognitivas.

Otro rumbo en la evolución de la evaluación es el que condujo a la evaluación de programas y a la evaluación institucional. En los años 60, la evaluación se comenzó a profesionalizar surgiendo nuevos pensadores sobre la evaluación educativa y, a raíz de ese fenómeno, en educación se plantea la necesidad de elaborar proyectos para el desarrollo del currículo; especialmente, en ciencias y matemática. Cronbach recomienda que se reconceptualice la evaluación "La evaluación es la colecta de informaciones con vista a la toma de decisiones" (CRONBACH, 1963, P. 674), por lo tanto esta dada: "como un proceso consistente en recoger y formalizar información que pueda ayudar a quienes elaboran los currículos" (RAma, 1989, P. 49).

Como hitos de la evaluación de esta época, es necesario destacar dos contribuciones por su influencia decisiva.

Los aportes de Cronbach en su artículo "Mejora de programas a través de la Evaluación” (1963), muestra la riqueza de las ideas propuestas en este estudio contribuyendo a dar otro rumbo a la evaluación. Se pueden citar de manera resumida las siguientes contribuciones:

- La evaluación como la obtención y uso de información para la toma de decisiones, mejoramiento del programa, necesidades y méritos de los estudiantes, y un reglamento sobre la calidad del sistema

- La necesidad de realizar evaluación del proceso y de la descripción de los productos.

- Los principios de la evaluación del aprendizaje no son enteramente apropiados para evaluar el mejoramiento de programas.

- La evaluación es una parte fundamental del desarrollo del currículo. 
- La evaluación colecta hechos que facilitarán el trabajo de mejoramiento del programa y hechos que permitirán una comprensión más profunda del proceso educacional.

- La evaluación debe identificar qué cambios produce un programa en el estudiante y qué aspectos necesitan revisarse. Las técnicas no deben limitarse a las pruebas de rendimiento.

- La evaluación debe comprender productos más generales yendo más allá del contenido del currículo, como los aspectos actitudinales, la aptitud para futuros aprendizajes y el potencial intelectual, entre otros.

- No debe haber criterios absolutos de comparación.

Por otro lado, en la misma época Scriven (1967) quien fue el primero en distinguir la evaluación formativa de la evaluación sumativa, propone:

- La evaluación tiene objetivos y cumple funciones.

- La evaluación debe evaluar objetivos y la medida en que estos se logran.

- Diferenciar la evaluación intrínseca de la evaluación extrínseca (efectos).

- Determinar el carácter comparativo de los estudios de evaluación.

Más tarde Scriven (1991) sostiene que la naturaleza de la evaluación no es la mera acumulación de datos que es relevante para la toma de decisiones sino que la evaluación cumple dos funciones; la de encargada de acumular datos y la de colecta, clarificación y verificación de valores relevantes y estándares.

Con estos aportes la evaluación no sólo se restringe a la evaluación del aprendizaje, sino que amplía su campo de aplicación abarcando los programas, además se señalan propósitos más generales, como por ejemplo la evaluación para la toma de decisiones, y se señala que la evaluación debe cumplir con dos funciones fundamentales la sumativa y la formativa, dándose importancia a la evaluación de proceso. Todas estas concepciones significan una apreciable evolución en el concepto de evaluación. 
El interés principal para Tyler como para Scriven son los resultados, en consecuencia su concepción de evaluación está orientada a la medición de la eficiencia y la productividad, elementos del punto de vista regulatorio.

Aún en la misma década, Stake (1967) propuso su modelo de evaluación, el modelo de comparación, que sigue la línea de Tyler. No obstante, su concepción es más completa al considerar las discrepancias entre lo que es observado y esperado y al formular hipótesis sobre las causas y las deficiencias en los resultados finales. Posteriormente casi 8 años más tarde este mismo autor se alejará de esta propuesta Tyleriana para postular algo más moderno sobre la evaluación y esta nueva propuesta radicará en que los objetivos del programa pueden ser modificados a través del tiempo, con el propósito de que el programa ofrezca una formación integral para responder a los problemas y cuestiones reales que son planteados por los participantes en función a sus expectativas.

Stake (1975, p.11) representaba su nueva propuesta de evaluación con la siguiente proporción:

Evaluación = Toda la constelación de valores de un programa Complejo de expectativas y criterios que distintas personas tienen sobre el programa

Según Stufflebeam y Shinfield (1987), este modelo exige un enfoque plural, flexible, interactivo, holístico, subjetivo y orientado al servicio. Este modelo hace énfasis en la evaluación de programas y considera importante la satisfacción de las expectativas de la persona que asiste al programa, es una referencia para una conceptualización posterior mencionada en la literatura, que se refiere a los estudiantes como clientes que hacen uso de un servicio educativo.

Stake manifiesta que la tarea básica del evaluador no es resolver la ecuación numéricamente ni, como recomendó Scriven, obtener un resumen descriptivo del programa. En cambio, Stake advierte al evaluador que realice un amplio informe de lo que se ha observado y de la satisfacción o insatisfacción que siente un grupo de personas adecuadamente seleccionadas frente al programa.

Influyó también en el desarrollo de la concepción de la evaluación el hecho de que otras ciencias como la adminis- 
tración y economía estaban muy interesadas en mejorar las formas de evaluación del proceso administrativo.

Entre las décadas de 1970 y 1980, la evaluación trata de responder a una diversidad de preguntas tales como: ¿qué es la evaluación?, ¿qué funciones tiene?, ¿cuál es el objeto de evaluación? Como era de suponer estas interrogantes encuentran diversidad de respuestas desde distintos enfoques y agentes. Es fácilmente comprensible que las exigencias que plantea la evaluación del aprendizaje por una parte y la evaluación de programas de otra, conduzcan a una gran variedad de esquemas y modelos evaluativos. Por otro lado se hace importante considerar los riesgos de la evaluación, así como la necesidad de implementar políticas considerando los resultados de la evaluación.

Guba y Lincoln (1989) hacen una propuesta de evaluación denominada respondente y constructivista, su planteamiento es que los resultados de la evaluación no son descripciones sino construcciones significativas elaboradas por los actores para dar sentido a las situaciones en las que se encuentran. Luego el carácter de la evaluación es fundamentalmente social, político, y orientado por valores.

Para la década de 1990, surgen nuevas innovaciones en los modelos de evaluación como resultado de nuevas necesidades de la evaluación del aprendizaje como institucional. Es iniciado un periodo de reflexión y de ensayos teóricos con voluntad de clarificar el proceso evaluativo. Estas reflexiones teóricas enriquecerán decisivamente el ámbito conceptual y metodológico de la evaluación, lo que unido a la enorme expansión de la evaluación de programas ocurrida durante estos años, dará lugar al nacimiento de esa nueva modalidad de investigación aplicada que hoy denominamos como investigación evaluativa.

Patton (2002, p.10) define la evaluación como "cualquier esfuerzo para aumentar la efectividad humana por medio de una investigación sistemática y basada en datos”, caracteriza su propio enfoque como una evaluación centrada en la utilidad buscada por los usuarios para usar los hallazgos en la toma de decisiones. Difiere de la investigación básica en que ésta busca generar o probar teorías para contribuir al conocimiento. 
Stufflebeam sumariza una serie de recomendaciones para llevar a cabo buenas investigaciones evaluativas y mejorar el sistema educativo. Según Escudero (2003), en estas recomendaciones, ya se observa el uso de conceptos importantes para la evaluación de instituciones educativas, tales como la evaluación de la calidad relacionada al valor y mérito de las instituciones, enfocando aspectos intrínsecos y extrínsecos entre los cuales se mencionan: el servicio para un contexto particular, la búsqueda de la mejora institucional en base a la evaluación, la evaluación del contexto, la observación de componentes formativos y sumativos, la evaluación de entradas, procesos y productos, el uso de métodos cuantitativos y cualitativos para analizar la información, la metaevaluación formativa y sumativa de los resultados de la evaluación. Como consecuencia de estos focos de influencia, se amplió considerablemente el fenómeno de la evaluación educativa. El sujeto directo de la evaluación siguió siendo el alumno, pero también todos aquellos factores que confluyen en el proceso educativo.

Para Stufflebeam (1998) hay conflicto entre los planteamientos del Joint Committee on Standards for Educational Evaluation con la corriente evaluadora denominada postmodernista representada por Mabry, Stake y Walker, Guba y Lincoln, según él existen muchos ámbitos de aproximación y el desarrollo de estándares de evaluación es perfectamente compatible con la atención a los diversos implicados, valores, contextos sociales y métodos. Stufflebeam defiende la colaboración en la mejora de las evaluaciones, estableciendo los estándares de manera participativa, pues cree que es posible la aproximación de planteamientos, con contribuciones importantes desde todos los puntos de vista.

La connotación política de la evaluación de programas e instituciones es mencionada por Carol Weiss, citada por Escudero en los siguientes términos:

No basta hablar del entorno social, es necesario hablar del entorno sociopolítico... En primer lugar, porque la investigación evaluativa trata con objetos, programas, instituciones, etc.; que son criaturas políticas, de impacto político. En segundo lugar, porque la evaluación se hace para alimentar 
la arena política, compitiendo con otros elementos y factores intervinientes en el proceso. Finalmente y en tercer lugar, por algo que según Weiss es menos reconocido, esto es, porque la propia evaluación tiene un sentido político en sí misma. (apud Escudero, 2005, p. 183)

House (2000) hace un análisis de diversos paradigmas de evaluación y sostiene que los enfoques de la evaluación se derivan de la filosofía del liberalismo, comparten ideas correspondientes a una sociedad mercantil, competitiva e individualista, la idea fundamental es la libertad de elección, su orientación es rotundamente empirista, asumen un mercado libre de ideas en el que los usuarios seleccionaran las mejores. Por otra parte da aportaciones para la realización de evaluaciones válidas. $\mathrm{Al}$ referirse a la validez de la evaluación de programas públicos afirma que debe ser veraz, creíble y correcta desde un punto de vista normativo. "La evaluación debe ser imparcial en el sentido que esten representados todos los intereses pertinentes" (House, 2000, P. 236).

\subsection{FUNDAMENTOS DE LA EVALUACIÓN EN LOS SISTEMAS DE EVALUACIÓN DE LA EDUCACIÓN SUPERIOR}

En América Latina los Modelos de Sistemas de Evaluación recogen en la concepción y procedimientos los aportes formulados por varios de los autores antes mencionados, por ejemplo Stufflebeam quien propone el uso varias perspectivas en la evaluación y métodos cualitativos y cuantitativos para análisis de la información.

\subsubsection{FUNDAMENTOS DE LA EVALUACIÓN EN EL SISTEMA NACIONAL DE EVALUACIÓN DE LA EDUCACIÓN SUPERIOR DE BRASIL}

El Sistema Nacional de Evaluación de la Educación Superior (Sinaes), puede ser interpretado como resultado de una evolución a partir de experiencias anteriores en la Evaluación de la Educación Superior, en la que es fundamental la contribución desde el ámbito académico. Dos experiencias anteriores con diferente concepción de la función de la evaluación que se dieron en la década de 1990 fueron: 
1) El Programa de Avaliação Institucional das Universidades Brasileiras (Paiub) que tuvo su base conceptual elaborada por la Associação Nacional dos Dirigentes das Instituições Federais de Ensino Superior (Andifes), con la contribución de notables investigadores en el área de evaluación. Pailharini resume las características del Paiub de la siguiente manera:

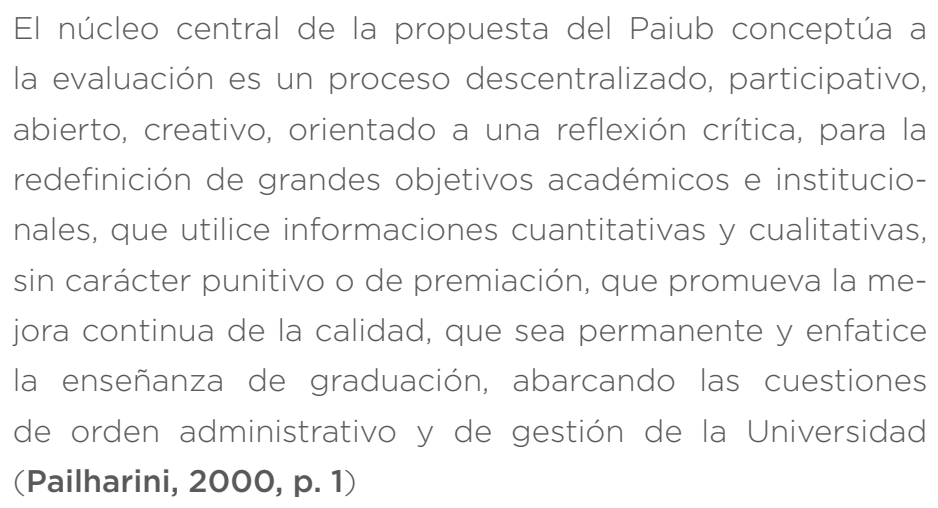

El Documento Básico resalta que la evaluación abarca tanto la autoevaluación como la evaluación externa, y que la participación de los miembros debe ser intensa en el proceso de evaluación con el propósito de integrar esfuerzos y experiencias existentes en la institución (BRASIL, 1993).

En la propuesta del Paiub pueden identificarse su afinidad con algunas contribuciones de:

- Crombach: Asociar el concepto de evaluación con la toma de decisiones para el mejoramiento del programa, la necesidad de realizar evaluación del proceso, no debe haber criterios absolutos de comparación, y las técnicas no deben limitarse a las pruebas de rendimiento.

- Guba y Lincoln: La evaluación es un proceso que implica colaboración y negociación, que permite comprender la situación en que se encuentran los actores. La evaluación es un proceso que orientado por valores.

- Stufflebeam: Las entidades educativas deben examinarse por su integración, servicio para un contexto particular, responsabilidades institucionales. La evaluación del proceso debe usarse para mejorar el plan de trabajo. Los estudios evaluativos se deben apoyar en la comunicación y en la inclusión sustantiva y funcional de los 
implicados. Los estudios evaluativos deben emplear múltiples perspectivas y métodos tanto cuantitativos como cualitativos para analizar la información.

2) El Examen Nacional de Cursos (ENC), conocido como Provão (1996). Este Examen era obligatorio para todos los alumnos formados en los programas o cursos escogidos por el Ministerio, iniciando esta evaluación con tres programas y acabó en el 2003 con 26 programas.

El Provão, tiene como fundamento concepciones de la evaluación diferentes a las de Crombach, Guba y Lincoln, Stufflebeam o House, remontándose su propuesta conceptual a las contribuciones de Tyler y Scriven de la evaluación de programas por comparación entre resultados y objetivos propuestos.

El año 2004, se instituyeel Sinaes, cuya concepción es presentada como: “... um modelo com visão integradora, abrangente, sistemática e participativa de avaliação”. En la cual se tiene como fuentes: la evaluación de las instituciones comprendiendo la evaluación interna (autoevaluación) y la externa, la evaluación de los programas de graduación (cursos) y los resultados del Examen Nacional de Desempeño de los Estudiantes (Enade), por lo tanto se constituye en una propuesta donde puede identificarse en la evaluación institucional y de programas, afinidad con elementosconceptualesdelascontribucionesdeCrombach,Gubay Lincoln y Stufflebeam de manera similar al Paiub y en la evaluación de estudiantes con Tyler y Scriven por priorizar el uso de indicadores de resultados en la evaluación. Por otra parte también la concepción de evaluación del Sinaes, recoge aportes conceptuales de investigadores en evaluación Brasileños que en el año2003formaron partedelacomisiónqueelaborólapropuestadel Sinaes; entre ellos mencionamos a:

- Dias Sobrinho (1995), quien define la evaluación institucional como "un emprendimiento sistemático, que busca la comprensión global de la universidad, por el reconocimiento y por la integración de sus diversas dimensiones" (apud Ristoff, 2003, P. 27). Es decir que para la evaluación se requiere políticas, estrategias, metas, decisión y voluntad de evaluados y evaluadores con el propósito de lograr la comprensión del objeto de la evaluación. Dias Sobrinho, amplia su concepción de evaluación al manifestar que: 
La opción para la evaluación como control (medida, balance, sentido dado, clasificación, selección, regulación, etc.) o como promoción de posibilidades (emancipación, mejora, formación, interpretación y producción de sentidos, construcción, dinamismo, etc.) resulta de distintas concepciones del mundo, que concurren entre sí, pero no se excluyen y, así mismo se complementan. (Dias Sobrinho, 2003, p. 39)

Esta concepción coincide con la propuesta de Sinaes en la cual coexisten las orientaciones de la evaluación regulatoria y formativa, y encuentra afinidad con lo propuesto por el francés Vial (1997, p. 41-42):

Por lo tanto, bajo una misma imagen, los evaluadores pueden asociar consecuentemente; el conflicto entre dos «logos», dos registros de palabras, dos «lenguajes»: el de Ratio ( «la evaluación debe ser justa, objetiva») y el de Pathos («evaluar es acompañar, cumplir, amar»).

También la dualidad en el carácter de la evaluación entre lo cualitativo y lo cuantitativo es mencionado por Dias Sobrinho cuando hace referencia a Barthes (autor holandés) manifestando que:

La evaluación es una reunión de valores, dice R. Barthes (1975:88). Por tanto es una acción filosófica, que incluye de modo radical la cuestión de los significados, los actos y los fines... En esa perspectiva, en cuanto reunión de valores y actitudes filosóficas, la evaluación es esencialmente cualitativa y subjetiva; interpreta y valora los fenómenos tomados como objeto. También es objetiva y cuantitativa; utiliza instrumentos y técnicas propios de los trabajos científicos con el fin de realizar análisis explicativos de los datos seleccionados. Esta forma objetiva y cuantitativa correspondería a la necesidad de facilitar y hacer confiable la información y presentación de resultados al público. (Dias Sobrinho, 2004, p. 8)

- Ristoff (2003) define a la evaluación como investigación que cumple funciones de ser sumativa, formativa, psicológica, sociopolítica y administrativa. Los objetos de evaluación son el alumno, docentes, técnicos, administrativos, aula, planes, programas de la disciplina, 
currículo, curso, Proyecto de Desarrollo Institucional, y hasta la misma institución. Debe tener métodos, criterios, procesos, etc. Se debe hacer evaluación sabiendo para qué y para quién servirá la información extraída. Y por último quien evalúa debe tener un nivel de profesionalismo, debe conocer sobre estadística, dominio de las relaciones humanas y debe conocer el interior de una universidad. Para completar con la definición se hace necesario que se haga evaluación de la evaluación, es decir meta evaluación en la que esta tenga características de utilidad, viabilidad, propiedad y exactitud.

En la concepción de Dilvo Ristoff se pueden identificar elementos conceptuales afines a las contribuciones de:

- Guba y Lincoln: La evaluación como proceso sociopolítico. La evaluación es un proceso que implica colaboración y negociación.

- Stufflebeam: Todo estudio evaluativo debe tener un componente formativo y otro sumativo. Los estudios evaluativos se deben apoyar en la comunicación de los implicados. Los estudios evaluativos deben ser evaluados, es decir se deben realizar metaevaluaciones.

- Isaura Belloni, quien define la evaluación institucional como:

... un emprendimiento que busca la promoción de la toma de conciencia sobre la institución. Su objetivo es mejorar la universidad. La autoconciencia institucional constituye importante subsidio para el proceso de la toma de decisión, tanto en el nivel individual cuanto en el nivel colectivo, de la institución como un todo, con vistas a su perfeccionamiento y tienen como punto fundamental de importancia la intensa participación de sus miembros tanto en la forma de encaminar la evaluación, en la identificación de criterios y procedimientos, como en la utilización de resultados. (apud Ristoff, 2003, p. 28)

En la concepción de Isaura Belloni, se pueden identificar elementos conceptuales afines a las contribuciones de:

- Crombach: Asociar el concepto de evaluación con la toma de decisiones para el mejoramiento. 
- Guba y Lincoln: La evaluación es un proceso que implica colaboración y negociación, que permite comprender la situación en que se encuentran los actores.

- Stufflebeam: Los estudios evaluativos se deben apoyar en la comunicación y en la inclusión sustantiva y funcional de los implicados.

Como se puede apreciar en las propuestas son identificables elementos conceptuales afines a las contribuciones de los clásicos de la Evaluación Norteamericana desde Tyler, Scriven, Crombach, Stufflebeam y Guba y Lincon, y Patton, inclusive los autores franceses y holandeses; quienes consideran que la evaluación está centrada en la utilidad buscada por los usuarios, estos aportes sirven de cimiento a la construcción de la concepción del Sistema Nacional de Evaluación para la Educación Superior (Sinaes), que se manifiesta como una concepción bipolar que abarca lo Regulatorio y lo Formativo, lo Cuantitativo y lo Cualitativo, y también lo Objetivo y lo Subjetivo.

\subsubsection{FUNDAMENTOS DE LA EVALUACIÓN EN EL \\ SISTEMA NACIONAL DE ACREDITACIÓN DE COLOMBIA}

El Sistema Nacional de Acreditación tiene como órgano de ejecución al Consejo Nacional de Acreditación (CNA). En el contexto Colombiano en el año 1992, se promulga la ley 30 con la cual se organiza el servicio público de la Educación Superior e instaura el CNA.

Para el Consejo Nacional de Acreditación:

La evaluación institucional supone el examen integral de la organización entera: abarca la misión y el proyecto institucional; la comunidad académica (estudiantes, profesores e investigadores); los procesos académicos (docencia, investigación, extensión o proyección social); el bienestar institucional; la pertinencia y el impacto social; los procesos de autoevaluación y autorregulación; la organización, la administración y la gestión; la planta física y los recursos de apoyo académico; los recursos financieros. (Colombia, 2010, p. 1)

De este modo la definición de evaluación expresa las dimensiones que abarca la evaluación institucional y la nece- 
sidad de contar con información cuantitativa y cualitativa para el proceso. Por otra parte:

La calidad de la educación superior es la razón de ser del Sistema Nacional de Acreditación; reconocerla, velar por su incremento y fomentar su desarrollo otorga sentido a la acción del Consejo Nacional de Acreditación. La calidad, así entendida, supone el esfuerzo continuo de las instituciones por cumplir en forma responsable con las exigencias propias de cada una de sus funciones. Estas funciones que, en última instancia, pueden reducirse a docencia, investigación y proyección social, reciben diferentes énfasis de una institución a otra, dando lugar a distintos estilos de institución.

(Aljure et al. 1998, p. 27)

Se señala que el concepto de calidad es fundamental en la propuesta del sistema de evaluación de la educación superior en Colombia y que esta en última instancia relacionada al cumplimiento de las funciones de la institución. A este respecto Uriel Giraldo, Darío Abad y Edgar Díaz, es su condición de miembros del CNA, señalan que:

Desde un punto de vista lógico se podrían establecer dos series de conceptos que cubran la noción de calidad.

Calidad como valor intrínseco de algo: mérito, excelencia, eficiencia.

Calidad como valor extrínseco de algo: rendimiento, pertinencia, eficacia.

Ambos conceptos son aplicables al objeto que trata de valuar la universidad y la enseñanza que imparte. En efecto, la educación superior desarrolla actividades que son valiosas por sí mismas: crear y distribuir conocimientos; educar, conservar y recrear la cultura, etc. Pero también produce talentos humanos, nuevas tecnologías, servicios que deben ser valorados en relación con las necesidades y las demandas de la sociedad. (Giraldo; Abad; Díaz, 2001, p. 11)

Los conceptos de calidad como valor intrínseco y extrínseco, son utilizados en el sistema de evaluación de Colombia, cuando plantean el nivel de excelencia en el logro de la calidad de las Instituciones de Educación Superior, y cuando plantean el mejoramiento y aseguramiento de la calidad con el uso de 
estándares que expresan el deber ser, y que permiten evaluar el ser y el hacer de programas e instituciones, considerando como dimensiones esenciales: la pertinencia, la eficacia y la eficiencia.

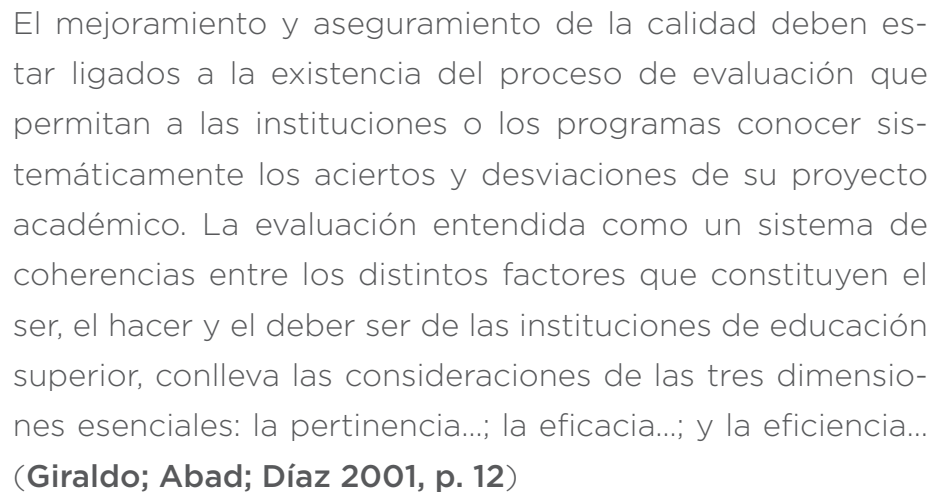

El énfasis en las coherencias entre los distintos factores que constituyen el ser, el hacer y el deber ser de las instituciones y su valoración en relación con las necesidades y las demandas de la sociedad, son afines a los aportes de Stufflebeam, quien también señaló que las entidades educativas deben ser valoradas tanto por su mérito (valor intrínseco, calidad respecto a criterios generales) como por su valor (valor extrínseco, y servicio para un contexto particular). La evaluación del proceso debe usarse para mejorar el plan de trabajo. Los estudios evaluativos se deben apoyar en la comunicación y en la inclusión sustantiva y funcional de los implicados. Los estudios evaluativos deben emplear múltiples perspectivas y métodos tanto cuantitativos como cualitativos para analizar la información.

Otro elemento conceptual afín a la propuesta de Stufflebeam el de establecer los estándares de manera participativa, buscando la aproximación de planteamientos, con contribuciones importantes desde todos los puntos de vista.

Por otra parte el propósito del sistema de evaluación es el mejoramiento de la calidad de las instituciones y programas, en el proceso se considera importante el conocimiento sistemático de los aciertos y desviaciones de su proyecto académico por lo que también puede identificarse afinidad con algunas contribuciones de:

- Crombach: Asociar el concepto de evaluación con la toma de decisiones para el mejoramiento del programa, y las técnicas no deben limitarse a las pruebas de rendimiento. 
- De Guba y Lincoln: La evaluación es un proceso que permite comprender la situación en que se encuentran los actores.

Otra preocupación en la propuesta de CNA es plantear un modelo de aseguramiento de la calidad, en el cual la calidad es hilo conductor en la articulación de un sistema de educación superior, pues el propósito es no sólo evaluar estándares y características de calidad y verificar su cumplimiento, sino asegurar su presencia y sostenibilidad en el desarrollo de la educación superior.

El sistema de acreditación dio origen al sistema de aseguramiento de la calidad que se apoya en el Sistema Nacional de Información de la educación superior, recibe la información del observatorio del mercado laboral acerca del desempeño laboral de los egresados que señala las necesidades del mercado y provee bases para la planeación prospectiva del sector.

En los conceptos anteriores extraídos de la propuesta del Consejo Nacional de Acreditación a partir de las contribuciones de sus miembros; se hace énfasis en que la evaluación institucional debe tener las siguientes características: debe comprender todos los componentes institucionales, debe tener métodos, criterios, procesos y propósitos claros. Los evaluadores deben tener la preparación requerida. Se debe buscar la comprensión global de la institución y de su entorno. Y además, es necesaria la toma de conciencia sobre la realidad institucional, para buscar el mejoramiento de las instituciones.

La evaluación institucional se enriquece con el recojo de datos cualitativos, que al ser analizados pueden ser transformados en resultados cuantitativos, por lo que el procesamiento de la información transita de lo cualitativo a lo cuantitativo, para obtener el conocimiento que permita mejorar: las instituciones, y el mismo proceso de la evaluación a través de la metaevaluación.

Luego también en el caso de Colombia se encuentran elementos conceptuales fundamentales para el diseño del sistema de evaluación que son afines a las contribuciones de los autores clásicos de evaluación norteamericanos, otros aportes procedentes de los autores de habla Hispana, es decir de España en mayor grado y de Cuba y México en menor grado, ha tenido mas eco en el sistema de educación escolar, en el que se formuló la formación orientada por competencias. 


\subsection{FUNDAMENTOS DE LA EVALUACIÓN EN LA COMISIÓN}

NACIONAL DE EVALUACIÓN Y ACREDITACIÓN UNIVERSITARIA

DE ARGENTINA (CONEAU)

En el caso de Argentina el organismo que cumple la función de realizar la evaluación institucional y de programas universitarios es la Comisión de Evaluación y Acreditación Universitaria, Coneau. Esta institución fue creada por la ley de educación superior 24.521, promulgada el 07 de agosto de 1995 y está en funcionamiento desde 1996.

Sin embargo desde inicio de la década de 1990 ya surge una notoria preocupación por la definición de calidad, promovida por el Consejo Interuniversitario Nacional (CIN), que buscó la transformación del Sistema de Educación superior influenciada directa e indirectamente por la creciente demanda de la iniciativa privada en ámbitos hasta entonces articulados por el sector público (GANDOLFo, 2008, P. 24).

Como consecuencia de este interés ya en 1993 el entonces Ministerio de Educación y Cultura de la Nación tenía firmados 16 convenios con universidades nacionales, dos con asociaciones de facultades y uno con una universidad privada, para planear e implementar procesos de evaluación institucional

En el marco legal en el que se crea la Coneau se señala que el proceso de evaluación debe respetar la autonomía universitaria:

El desarrollo del concepto de evaluación en nuestro país debería apuntar a medir el sentido y la significación personal y social de las actividades y las prácticas, y por el correlato entre los fines educativos y las necesidades sociales e históricas de la comunidad argentina. Requisito imprescindible es el de la Autonomía... La evaluación de la calidad, entonces, surge de una reflexión sobre los procesos de crecimiento interno de las instituciones universitarias, que valore las transformaciones en una confrontación permanente con la realidad y las intencionalidades de los sujetos, que construyen y reconstruyen permanentemente sus proyectos (Argentina, 1992, p. 12).

La autonomía es concedida en la Ley de Normalización Universitaria en 1984, es redefinida en la Ley Federal de Educación de 1993, según Gandolfo (2008, p. 28) “Por la ley 24.521 la autonomía universitaria fue redefinida y acoplada directamente a un concepto de calidad que parece apoyarse fuertemente en un 
modelo técnico-objetivo a la vez que cargado de poder", y tuvo reconocimiento constitucional a partir de la constitución de 1994.

La importancia de evaluar la calidad en el proceso, se refleja en el acuerdo número 50, emitido por el CIN en 1992, que dice:

Considerando que la evaluación de la calidad universitaria constituye un instrumento de singular significación en la gestión universitaria y que la Comisión de Enseñanza ha realizado diversas reuniones con los Secretarios Académicos de las Universidades Nacionales y con la Comisión de Seguimiento y Análisis del Subproyecto 06, se acuerda: Art. 10: Definir como Aspectos Relevantes de la Evaluación de la Calidad Universitaria... (Argentina, 1992, p. 12)

La Coneau de Argentina al igual que Colombia parte desde la evaluación institucional, sin embargo para el caso argentino los fundamentos de evaluación que orientan el sistema no se construyen a través de investigaciones, muy por el contrario; sino a través de un trabajo de reflexión y discusión en reuniones en CIN, a través de diálogos entre los miembros del consejo con los asesores para encaminar las acciones del proceso de evaluación institucional. Con relación a la definición de la evaluación institucional se menciona:

En esta reflexión partimos de pensar que la evaluación institucional supone un proceso complejo para determinar el valor de algo, que implica una delicada tarea de interpretación de un conjunto de elementos que interactúan configurando una realidad particular y significativa. De hecho, evaluar no es, rigurosamente la etapa posterior o final de un proceso, sino un momento en el camino para apreciar lo caminado, y decidir cómo continuar. Si se sigue profundizando, se puede decir que la evaluación que se formula debe aspirar a la credibilidad y al consenso. Una evaluación, asimismo, no es los "datos", aunque éstos sean indispensables, sino el proceso por el que se aprecia y discierne el valor de las acciones y realizaciones; un proceso profundamente humano que se nutre y se articula en el diálogo, la discusión y la reflexión. (Argentina, 1997, p. 6)

En los informes memoria se puede apreciar los aspectos importantes relacionados con la propuesta de evaluación institucional y acreditación: 
La evaluación y la acreditación, tareas específicas de esta Comisión, no debieran verse como destinadas a frenar todo crecimiento o a bloquear la pluralidad. La búsqueda de la calidad, hoy más que nunca, admite y promueve la diversidad organizativa y la sana competencia entre instituciones. Se trata más bien de potenciar esa búsqueda, de modo que las instituciones se vean estimuladas a alcanzar metas de desempeño cada vez más altas, a representarse a sí mismas, a admitir que la mirada externa es conveniente y también legítima. A instalar, en suma, una cultura de la evaluación. (Argentina, 1996, p. 7)

En relación a la evaluación de la calidad se menciona que es importante el análisis de las características estructurales de la universidad, de sus misiones y sus funciones, Astin (1990) señala que un valor fundamental de la evaluación consiste en la posibilidad de que a través de ella una institución se entienda realmente a sí misma, conozca sus fortalezas y sus debilidades, sus limitaciones y potencialidades. Mediante tal conocimiento la institución está más cerca de lograr éxito que otra que carece de dicho autoconocimiento. Resaltando que para ello es importante la autonomía universitaria. En la evaluación se propone el análisis y la valoración de los procesos y resultados alcanzados respecto a los programas y proyectos contenidos en sus planes de desarrollo institucional.

Evaluaciones externas: consisten en el análisis de las dimensiones y logros del proyecto de la institución universitaria en el marco de sus misiones y objetivos. Se hacen como mínimo cada seis años y se llevan a cabo en el marco de los objetivos definidos por cada institución. Son complementarias de las autoevaluaciones que las instituciones disponen para analizar sus logros y dificultades y para sugerir medidas para el mejoramiento de la calidad universitaria. Las evaluaciones externas tienen como objetivo principal asistir a las instituciones en las propuestas de mejoría de la calidad y conducen a emitir recomendaciones públicas a este respecto. (Argentina, 1996. p. 9)

La propuesta de evaluación institucional argentina, fue receptiva de contribuciones de otras experiencias anteriores de 
sistemas de evaluación de la educación superior en América Latina. La CNA de Colombia figura en actas de reuniones de los miembros de la Coneau, como órgano asesor.

La importancia de dar prioridad al autoconocimiento de las organizaciones por los miembros de las organizaciones se señala en los Lineamientos de la Coneau,

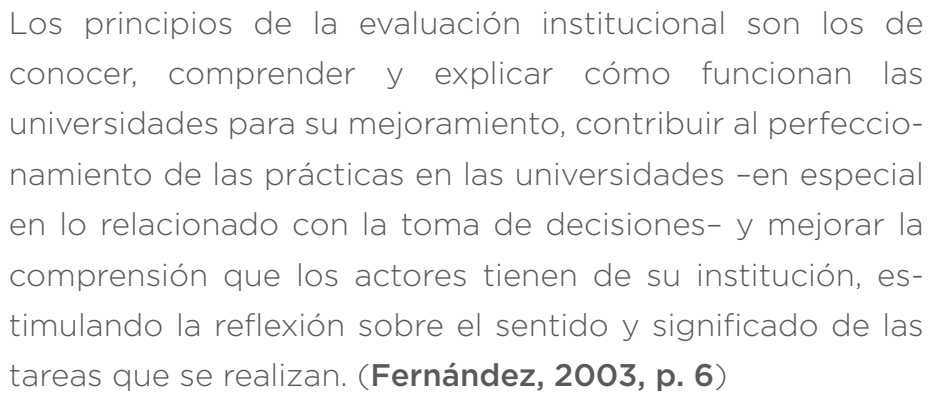

La necesidad de que las instituciones respondan a una realidad histórica y contextual, es mencionada por Pérez Rassetti (2009), quien señala que el modelo que cada universidad se propone está en el proyecto institucional, por lo tanto: “... mejorar, objetivo de la evaluación institucional, es ajustar ese modelo a la realidad histórica y contextual y recorrer un camino que achique la distancia entre el modelo revalidado y la realidad institucional" (PÉREZ, 2009, P. 2).

En la propuesta de la Coneau pueden identificarse afinidad con los aportes de

- Guba y Lincoln: los resultados de la evaluación no son descripciones sino construcciones significativas elaboradas por los actores para dar sentido a las situaciones en las que se encuentran. La evaluación es un proceso que implica colaboración y negociación.

- Stufflebeam: Las entidades educativas deben examinarse por su integración, servicio para un contexto particular, responsabilidades institucionales. La evaluación del proceso debe usarse para mejorar el plan de trabajo. Los estudios evaluativos se deben apoyar en la comunicación y en la inclusión sustantiva y funcional de los implicados. Los estudios evaluativos deben emplear múltiples perspectivas y métodos tanto cuantitativos como cualitativos para analizar la información. 
- Crombach: Asociar el concepto de evaluación con la toma de decisiones para el mejoramiento del programa. No debe haber criterios absolutos de comparación. Las técnicas no deben limitarse a las pruebas de rendimiento.

\section{CONCLUSIONES}

Ralph Tyler hizo un aporte tecnicista a la evaluación del aprendizaje como medición, resaltando la importancia de los objetivos, técnicas e instrumentos para evaluar el comportamiento de los estudiantes en el proceso de enseñanza-aprendizaje.

Con los aportes de Cronbach, Scriven y Stake, la evaluación no se restringe a la evaluación del aprendizaje, sino que amplía su campo de aplicación abarcando la evaluación de programas, además los aportes mencionan propósitos más generales: la evaluación para la toma de decisiones, las funciones de la evaluación la sumativa y la formativa, dándose importancia a la evaluación de proceso.

Stufflebeam, da aportes sustanciales para la aplicación de la evaluación a las instituciones educativas, tales como la evaluación de la calidad relacionada al valor y mérito de las instituciones, enfocando aspectos intrínsecos y extrínsecos entre los cuales se menciona el servicio para un contexto particular. La búsqueda de la mejora institucional en base a la evaluación. La evaluación del contexto. La observación de componentes formativos y sumativos. La evaluación de entradas, procesos y productos. El uso de métodos cuantitativos y cualitativos para analizar la información. La metaevaluación formativa y sumativa de los resultados de la evaluación.

En América Latina los investigadores sobre evaluación tomaron como referencia a los autores anteriormente mencionados, realizando reconceptualizaciones al aplicar la evaluación a las instituciones educativas haciendo énfasis en la calidad, y distinguiendo aspectos formativos y regulatorios, así como la necesidad de comprender el funcionamiento de las instituciones, y la toma de consciencia de los miembros para la búsqueda del mejoramiento de las instituciones.

El enfoque comparativo aplicado a la evaluación institucional en Latinoamérica, permite descubrir similitudes que 
pueden fortalecer las propuestas de evaluación tales como: la necesidad de mejora de calidad de las instituciones con respecto de su autonomía, los resultados de la evaluación tienen un carácter relativo a cada institución y es necesario desarrollar sistemas de evaluación que contribuyan al fortalecimiento de los sistemas de educación nacionales.

\section{REFERÊNCIAS BIBLIOGRÁFICAS}

ALJURE, Nasser Emilio et al. La Evaluación externa en el contexto de la acreditación en Colombia. 1998. Disponible en: <http://www.cna.gov.co/1741/ articles-186502_eval_ext_en_colombia.pdf>. Accesado en: 9 mar. 2010.

ASTIN, A. W. Assessment as a tool for institutional renewal and reform. In: AMERICAN ASSOCIATION FOR HIGHER EDUCATION ASSESSMENT FORUM, ASSESSMENT 1990: Accreditation and Renewal, AAHE, Washington, D.C., p. 19-33. 1990.

ARGENTINA. Comisión Nacional de Evaluación y Acreditación Universitaria. Memoria Anual, 1996.

. Comisión Nacional de Evaluación y Acreditación UniversitariaCONEAU. Lineamientos para la evaluación institucional, Aprobado por Resolución Nº 094-CONEAU-1997.

Consejo Interuniversitario Nacional, Acuerdo № 50/1992, Adhesión al Acuerdo Del CIN No 50/92.

BRASIL, Ministério da Educação. Avaliação das universidades brasileiras. Secretaria de Educação Superior. Comissão Nacional de Avaliação: documento básico. Brasília: SESu, 1993.

BROADFOOT, P. Assessment, schools and society (Contemporary Sociology of the School). London: Methuen, 1979.

CASTRO PIMIENTA, Orestes. Evaluación integral del paradigma a la práctica. La Habana. Cuba: Pueblo y Educación, 1999.

COLOMBIA, Consejo Nacional de Acreditación, La Calidad en la acreditación institucional. 2010. Disponible en: <http://www.cna.gov.co/1741/ article-190811.html> . Accesado en: 21 feb. 2010.

CRONBACH, Lee. J. Course improvement through evaluation. Teachers College Record, v. 64, n. 8, 1963, p. 672-683.

DIAS SOBRINHO, José Dias. Avaliação da educação superior, regulação e emancipação. In: SOBRINHO, José Dias; RISTOFF, Dilvo. Avaliação e compromisso público. Florianópolis: Insular, 2003, p. 35-52.

. Autonomía y Evaluación. Revista Reencuentro. México, v. 8, n. 40, 2004. Disponible en: <http://redalyc.uaemex.mx/redalyc/src/inicio/ArtPdfRed. jsp?iCve=34004003\&iCveNum=5520>. Accesado en: 10 enero 2010. 
ESCUDERO ESCORZA, Tomás. Claves identificativas en la evaluación evaluativa: Análisis desde la práctica. Contextos Educativos. España, v. 8, n. 9, 2005, p. 179-199. Disponible en: <http:/www.unirioja.es/servicios/sp/ej/contextos/infos/2091397. pdf>. Accesado en: 14 dic. 2009.

. Desde los tests hasta la investigación evaluativa actual. Un siglo, el XX, de intenso desarrollo de la evaluación en educación. Revista Relieve. v.9, n.1, 2003, p.11-43. Disponible en: <http://www.uv.es/RELIEVE/v9n1/ RELIEVEv9n1_1.pdf>. Accesado en: 12 dic. 2009.

FERNANDEZ LAMARRA, Norberto. Evaluación y acreditación en la educación superior argentina. In: SEGUNDO CONGRESO ARGENTINO DE ADMINISTRACIÓN PÚBLICA, SOCIEDAD, ESTADO Y ADMINISTRACIÓN. 2003. Disponible en: <http://www.aaeap.org.ar/ponencias/congreso2/Fernandez Norberto.pdf>. Accesado en: 12 oct. 2010.

FERRÁN JULIÁ, Ferrer. La Educación comparada actual. Barcelona: Ariel, 2002.

GANDOLFO, Fabio Hugo. La Garantía de calidad para la universidad pública Argentina a partir de la década del 90. Una aproximación a la confrontación de modelos en el campo de la autonomía. In: ENCUENTRO NACIONAL, 5 Y LATINOAMERICANO, 2: La Universidad como objeto de investigación. Buenos Aires: Universidad Buenos Aires, p. 21-34. 2008.

GIRALDO, Uriel; ABAD, Dario; DÍAZ, Edgar. Bases para una política de calidad de la educación superior en Colombia, In: CONSEJO NACIONAL DE ACREDITACIÓN. Investigaciones. Colombia, 2001.

GUBA, E. G.; LINCOLN, Y. S. Fourth generation evaluation. Newbury Park, California: Sage, 1989.

HOUSE, Ernest R. Evaluación, ética y poder. Madrid: Editora Morata, 2000.

MELO, Claudia Sofía. Filosofía y Educación en los Años 80 y 90. In: LECHUGA, Martínez, Susana. Reseña de la evaluación por competencias: matemáticas, ciencias sociales, filosofía. Evolución de las pruebas de Estado. México. v. 5, n. 10. 2004.

MIGNONE, Emilio F. Acreditación. Trabajo anterior a la Ley 24.521. 1995.

PAILHARINI, Francisco de Asis. O Paiub em universidades federais da região sul e sudeste: tormento ou paixão. In: ASSOCIAÇÃO NACIONAL DE PÓS-GRADUAÇÃO E PESQUISA EM EDUCAÇÃO, p.1-16. 2000. Disponible en: <www.anped.org.br/ reunioes/23/textos/1117t.PDF>. Accesado en: 11 feb. 2010.

PATTON, Michael Quinn. Qualitative evaluation and research methods. Sage Publications, 3ra. Edición. Newbury Park, CA. 2002.589 p. Disponible en: <http://www.amazon.com/Qualitative-Research-Evaluation-Methods-Michael/ dp/0761919716\#reader_0761919716>. Accesado en: 15 mar. 2011.

PÉREZ RASETTI, Carlos. Cuestiones teóricas y metodológicas de evaluación y acreditación. UNILA. Brasil. 2009.

PINAR, W. et al. Understanding curriculum. New York: Peter Lang. 1995.

RAMA, Germán. Cambio social, educación y crisis en América Latina. Análisis, n. 49-50, enero-diciembre, 1989.

RISTOFF, Dilvo I. Algunas definiciones de avaliação. In: DIAS SOBRINHO, José; RISTOFF, Dilvo I. Avaliação e compromisso público, Santa Catarina, Brasil. 
Editora Insular, 2003, p. 21-33.

SCRIVEN, M. Evaluation thesaurus, $4^{\text {th }}$ ed. California: Sage, 1991.

The Methodology of evaluation. In: TYLER, R.W.; GAGNE, R. M.; SCRIVEN, M. Perspectives of curriculum evaluation. Chicago: Rand McNally, p. 39-83, 1967. (AERA Monograph 1.)

STAKE, R. E. Program Evaluation, Particularly Responsive Evaluation. CIRCE, University of Illinois at Urbana-Champaing, November, 1975. Disponible en: <http://www.globalhivmeinfo.org/CapacityBuilding/Occasional\%20 Papers/05\%20Program\%20Evaluation,\%20Particularly\%20Responsive\%20 Evaluation.pdf $>$. Accesado en: enero 2010.

The Countenance of educational evaluation. Illinois: CIRCE,

University of Illinois at Urbana-Champaign, v. 68, n. 7, p.523-540, 1967.

STUFFLEBEAM, D. L. Conflicts between standards-based and postmodernist evaluations: Toward rapprochement. Journal of Personnel Evaluation in Education, v. 12, n. 3, 1998, p. 287-296.

STUFFLEBEAM, D. L.; SHINFIELD, A. J. Evaluación sistemática, guía teórica y práctica. Madrid: M.E.C; Paidós Ibérica, 1987.

TYLER, Ralph W. Basic principles of curriculum and instruction, suspected that his little book of only eighty-three pages would make such an indelible mark on the field of curriculum theorizing, as well as on teaching practices in the American public schools. 1949.

VIAL, Michael. Préface: Essai sur le processus de référenciation l'evaluateur en habits de lumière In: BONNIOL, Jean-Jacques; VIAL, Michel. Les Modèles de l'évaluation: textes fondateurs avec commentaires. Bélgica: Boeck \& Larcier, p. 21-46, 1997.

\section{NINA ELEONOR VIZCARRA HERLES}

Doctorado en Administración de la Universidad Estatal Federico Villareal de Lima, Peru, Post doctoranda de la Facultad de Educación de la Unicamp herleseleonor@yahoo.com.br

\section{ELISABETE MONTEIRO DE AGUIAR PEREIRA}

Doctorado en Educación en la Universidad Estatal de Campinas - Unicamp, profesora libre docente de la Facultad de Educación de la Unicamp eaguiar@unicamp.br 